Ganz besonders im Bereich der Impfungen ist es für den mündigen und interessierten Konsumenten wichtig, sich auf Informationen abstützen zu können, die hinsichtlich Neutralität, Objektivität und Wahrheitsgehalt eine gute Basis für einen informierten Entscheid darstellen.

Begrüssenswert ist einerseits, dass der Konsumentenschutz sich dieser heiklen Thematik annimmt und dazu die Broschüre «Impfen - Grundlagen für einen persönlichen Impfentscheid» herausgibt.

Andererseits stellen sich Fragen: Sind die Aussagen in der Broschüre effektiv objektiv? Sind die medizinischen Impfinformationen wissenschaftlich korrekt? Und letztendlich: Kann die Leserin/der Leser nach der Lektüre dieser Broschüre tatsächlich eine informierte Wahl treffen?

Die nachstehenden Stellungnahmen dreier Fachgesellschaften zeigen deutlich, dass die vorliegende Broschüre minimale Voraussetzungen nicht erfüllt, die bei einer derart wichtigen Fragestellung notwendig sind. Zu hoffen bleibt, dass bei der Erarbeitung der 7. Auflage auch anerkannte, glaubwürdige Fachpersonen zu Rate gezogen werden und die Broschüre dem Konsumenten auch einen tatsächlichen Dienst erweisen kann.

Ressort Gesundheit und Prävention FMH

\title{
Stellungnahme zur Broschüre «Impfen - Grundlagen für einen persönlichen Impfentscheid» des schweizerischen Konsumentenschutzes (6. Auflage)
}

\section{Société Suisse de Pédiatrie (SSP/SGP)}

L'analyse différenciée de cette brochure nous mène à la position suivante:

Le message sous-tendu tout au long de la brochure est toujours empreint d'une trop grande retenue, d'une trop extrême prudence qui déconseille toute intervention, voire même d'une méfiance persistante face à l'attitude des recommandations officielles médicales et politiques pourtant basées sur des données internationalement reconnues comme valides.

Les assertions dans la brochure ne sont pas correctes et ne permettent pas de faire un choix éclairé. Ceci notamment car il manque régulièrement une pondération numérique dans l'énumération des risques naturels des maladies et des effets secondaires des vaccinations. Par analogie, on pourrait imaginer une brochure décrivant sur un même plan les plaisirs et bienfaits des sports ou des vacances et les risques d'y contracter des maladies et d'y subir des accidents (ceci donnerait par analogie: «les vacances en Grèce peuvent être dangereuses parce qu'un enfant ayant développé une leucémie s'était tapé la tête au fond d'une piscine en Grèce des années auparavant»).

En outre, la brochure transmet des informations fausses entre autres:
- Sur la biologie de l'immunité: «il n'y a pas de pression du temps à vacciner tôt (p. 5)». Au contraire, il est formellement établi que les anticorps transmis par la mère décroissent de moitié toutes les 3 semaines et qu'en l'absence de vaccination l'âge moyen des nourrissons présentant une méningite à Hib ou à pneumocoques est de 4-5 mois (avec ou sans allaitement)! Pour les prématurés, la situation est encore plus urgente, puisqu'ils naissent avec des taux d'anticorps maternels beaucoup plus faibles. Enfin, la majorité des enfants hospitalisés avec une coqueluche ont moins de 6 mois ...

- Sur la virologie: il n'y a jamais eu aucune relation immunologique susceptible de permettre une protection «croisée» entre le virus respiratoire synctial (RSV) et le virus de la rougeole (p. 25); l'état immunitaire des mères envers la rougeole n'a jamais eu et n'aura donc jamais aucune influence sur la fréquence des bronchiolites à RSV, qui ont toujours atteint les petits enfants et continueront de le faire tant qu'un moyen efficace de prévention ne sera pas disponible! L'augmentation des déclarations à l'OFSP est liée à l'introduction des tests de diagnostic et 
celle des hospitalisations reflète l'introduction des mesures transcutanées d'oxymétrie et de la recommandation d'hospitaliser les enfants dès l'atteinte d'une saturation inférieure à $94 \%$ et non plus seulement à $90 \%$...

- Sur l'autisme: la prétendue relation entre la présence de mercure et l'augmentation massive de l'autisme (p. 11) a été largement recherchée depuis ces allégations, sans toutefois être confirmée par les études contrôlées.

- Sur le retrait du vaccin Hexavac ${ }^{\circledR}$ : il a été clairement établi communiqué par les autorités responsables des produits de santé (EMEA en Europe, Swissmedic en Suisse) que l'Hexavac ${ }^{\circledR}$ était retiré du marché en raison d'incertitudes quant à son immunogénicité à long terme résultant de modifications dans les processus de production et non l'existence de morts subites survenues dans les jours suivant une vaccination (p. 12).

- La prévention du tétanos en cas de blessure: la prévention du tétanos par injection de sérum (p. 37) berce d'illusions: les blessures dangereuses passent souvent inaperçues car il suffit d'une blessure peu étendue (piqure, ...) ne conduisant donc pas à la demande d'une prévention par immunoglobulines. Et s'il y a un geste dont les scientifiques ont appris à se méfier, c'est bien celui des injections à l'étranger (risque de transmission d'hépatites B et/ou C ou de VIH par utilisation de matériel non stérile) d'un sérum d'origine humaine (risques de contamination encore inconnues, comme les prions).

La brochure véhicule aussi des informations pseudo-scientifiques dans le sens où elle prétend (p. 13) que des maladies autrefois bénignes seraient considérées maintenant comme dangereuses. Non, la rougeole et ses encéphalites n'ont jamais été bénignes. Non, le cancer du col de l'utérus ne l'est pas. C'est le développement des connaissances et l'augmentation des exigences de santé de la part de la population, parallèlement à l'augmentation du niveau de vie, qui rendent ces maladies inacceptables $\mathrm{au} \mathrm{XXI}^{\mathrm{e}}$ siècle et nous engagent dans la voie de la prévention par la vaccination, lorsque celle-ci devient possible...

Le chapitre sur la rougeole (p. 46-47) contient toujours de nombreuses inexactitudes et contrevérités:

- Il reconnaît que (grâce à la vaccination!) la rougeole ne survient plus en Suisse que dans des épidémies limitées, qu'il traite de «petites et locales». En 2003, ce sont pourtant plus de 670 cas qui ont été déclarés à l'OFSP, en provenance de 20 cantons ...
- Il indique que le risque de la complication la plus grave (la panencéphalite subaiguë progressive ou SSPE) est plus élevé chez les nourrissions de mères vaccinées qui s'infectent dans la première année de vie dès la perte des anticorps maternels ... sans dire que le risque est bien supérieur chez les nourrissons de mères non vaccinées! Le dernier enfant décédé de SSPE à Genève, il y a seulement quelques années, avait été contaminé par la rougeole de sa mère juste avant sa naissance ...

- Il indique qu'un vaccin monovalent contre la rougeole est disponible en Suisse, ce qui n'est actuellement plus le cas.

- Il indique que l'élimination de la rougeole nécessite une couverture vaccinale de 95\% qui ne serait pas atteignable ... sans citer les nombreux pays qui ont déjà réussi à éliminer la rougeole de leur territoire.

- Il indique que l'encéphalite après vaccination contre la rougeole pourrait survenir dans un cas sur 11000 - ce qui est tout à fait incompatible avec les données de surveillance qui indiquent un risque maximal (non confirmé!) de 1 par million.

- Il indique la suspicion d'un lien avec les maladies inflammatoires chroniques de l'intestin et l'autisme sans indiquer que toutes les études contrôlées ont confirmé l'absence d'association entre vaccination ROR et ces deux pathologies.

- Il continue à mentionner que le fait de ne pas faire des «maladies d'enfance» augmenterait le risque de cancer, sclérose en plaques et autres défauts de l'immunité ...

Le chapitre sur les méningocoques (p. 54-55) réunit plusieurs défauts:

- La recommandation suisse actuelle n'est pas limitée à des groupes à risque particuliers.

- Il n'y a pas de vrai «paradoxe anglais» puisque la mortalité rapportée est due au méningocoque du groupe $\mathrm{B}$ et non au groupe $\mathrm{C}$ seul contenu dans le vaccin.

- Les réactions locales sont certes fréquentes, mais il est faux de les mettre sur le même plan de probabilité que les réactions générales.

- Les 6 décès britanniques rapportés sont à mettre malheureusement en relation avec les morts subites du nourrisson, dont le nombre global n'a pas augmenté! Il serait aussi honnête de signaler que cette coïncidence ne nous concerne pas puisque ce vaccin n'est recommandé par l'OFSP qu'après l'âge de 12 mois.

- Des cas de syndrome de Guillain-Barré ont été rapportés en association temporelle lors de l'introduction d'un vaccin quadrivalent conjugué (non encore disponible en Suisse!) 
dans les collèges américains. Le suivi par le CDC a montré qu'il s'agissait d'une fausse alerte et écarté une relation causale entre vaccination et Guillain-Barré.

Le chapitre sur les pneumocoques (p. 56-57) instille lui-aussi des informations mal pondérées qui désorientent les lecteurs-trices:

- Il oppose de façon occulte les 90 sérotypes de germes naturels aux quelques 7 ou 23 sérotypes visés par les vaccins, sans mentionner que les 7 proposés représentent environ $80 \%$ des souches responsables des infections invasives des petits enfants en Suisse.

- Certes le naso-pharynx se recolonise, mais avec des germes moins pathogènes et moins résistants aux antibiotiques, si bien que le risque d'infection invasive (méningite, bactériémie) est considérablement diminué (>90\%) par la vaccination.

- Les effets secondaires sont décrits sans distinguer ceux qui pourtant classiquement attribués au vaccin DTPa administré concomitamment (pleurs persistants, hypotoniehyporéactivité) et non à la vaccination contre les pneumocoques; ils mettent à nouveau sur le même plan la fièvre, assez courante, et les convulsions, très rares.

Ce qui nous mène à la conclusion suivante:

- Il s'agit d'une brochure rédigée à dessein d'une manière subtile en utilisant systématiquement des éléments inquiétants sans la pondération nécessaire et délivrant ainsi un message tellement méfiant qu'on ne peut aucunement la qualifier de neutre.

- On ne peut que déconseiller cette brochure qui n'est pas un outil crédible permettant de soutenir un choix éclairé des parents soucieux d'une information de qualité au moment de faire des choix aussi importants que ceux concernant la vaccination de leurs enfants.

Dr Pierre Klauser Past Président SSP/SGP Spécialiste FMH en Pédiatrie

92, rte de Frontenex 1208 Genève

\section{Schweizerische Gesellschaft für Infektiologie}

Die Voraussetzungen für einen neutralen «Ratgeber» sind in der vorliegenden Broschüre eindeutig nicht gegeben. Um nicht auf jede einzelne Seite des Ratgebers einzugehen, wird die Stellungnahme im spezifischen Teil anhand des Beispieles Keuchhusten ausgeführt.

\section{Allgemeines}

Positive Punkte:

- Der Konsument soll informiert werden

- Der Konsument soll geschützt werden

- Bekennung zu Objektivität und ethischem Handeln als Ziel

- Freundliche, ansprechende und leserfreundliche Aufmachung

- Für den Laien verständliche Sprache

- Gutes Bildmaterial

- Keuchhusten: Darstellung der Krankheit inhaltlich korrekt

Negative Punkte:

- Hält sich überhaupt nicht an den im Hinweis zur 6. Auflage angeführten Satz «Entsprechend basiert dieser Ratgeber auf einer sorgfältigen, differenziert-kritischen Auseinandersetzung mit dem Für und Wider von Impfungen, überlässt den konkreten Entscheid der Leserin und dem Leser». So wird z.B. schon im ersten Absatz des Vorwortes eine Interpretation zum Titel der aktuellen Impfbroschüre des BAG geliefert und daraus dem BAG eine für den Konsumenten negative Haltung unterstellt.

- Ebenfalls schon im Vorwort werden Behörden, Ärzte und Industrie gegenüber kritischen Ärzten und Konsumentenorganisationen nicht mit objektiven Kriterien klassiert und einander gegenübergestellt, so fehlt z.B. die Definition von «kritischem Arzt».

- Suggeriert auch bereits im Vorwort, dass Behörden und Ärzte eine «ethische Aufgabe» nicht wahrnehmen.

- Verwendung von Schlagwörtern, ähnlich wie bei Werbetexten, also wovor der Konsumentenschutz üblicherweise warnt und wogegen er sich auch - berechtigterweise - wehrt.

- Fehlende Balance in der Beschreibung von Interessen der einzelnen Exponenten und in den farblich hervorgehobenen Kommentaren.

- Unzählige Ungereimtheiten und widersprüchliche Angaben bzw. Äusserungen oder Interpretationen.

Beispiel Keuchhusten:

- Nicht erwähnt wird, dass Keuchhusten weltweit 300000 Todesfälle pro Jahr fordert und dass er zu den zehn häufigsten Todesursachen im Kindesalter gezählt werden muss, hauptsächlich bei nicht geimpften Kindern (Lancet Infect Dis 2003;3:413-8 und Lancet 2006; 367:1926-36). Die Angabe 0,1\% zur Todesrate bei Keuchhusten im Säuglingsalter ist nicht richtig; die Literaturangaben bewegen sich 
zwischen 0,1 und 1\% (Pediatrics 1997;100: e10).

- Der Abschnitt über die Impfung enthält Unstimmigkeiten: Richtig wird festgehalten, dass junge Säuglinge nicht in ausreichendem Mass vom Impfschutz der älteren Geschwister profitieren, weil andere Infektionsquellen, nämlich Jugendliche und Erwachsene, bislang nicht von den Impfempfehlungen erfasst sind. Nicht korrekt ist, deswegen aber die Geschwister auch nicht zu impfen. Dies würde ja noch mehr Keuchhustenfälle bei jungen Säuglingen erwarten lassen und wäre demnach kontraproduktiv. Entsprechend ist die Aussage irreführend, dass die Auffrischimpfungen im Vorschul- und Schulalter nur einen «bescheidenen zusätzlichen Nutzen» bringen. Zum einen werden die Geimpften selbst dadurch besser geschützt, zum anderen trägt dies zur Herdenimmunität bei.

- Die Ansteckung von Säuglingen durch Eltern ist kein neues Phänomen und wurde schon lange vor Einführung der Pertussis-Impfung berichtet (Med Klinik 1921;17:1173 und Med Klinik 1921;17:1447-1448). Also ist dies sicher nicht die Folge der Einführung der Keuchhusten-Impfung wie im «Ratgeber» geschrieben.

- Der Abschnitt Wirksamkeit und Nebenwirkungen der Impfung enthält wiederum Unstimmigkeiten: «Schutzwirkung des Ganzkeimimpfstoffs nur sehr ungenügend» - das ist nicht korrekt, die ermittelten Schutzraten in den zahlreichen Wirksamkeitsstudien der früheren 90er Jahre ergaben - mit Ausnahme eines spezifischen Produktes - Schutzraten von $\geq 90 \%$ (Textbook of Pediatric Infectious Diseases. $5^{\text {th }}$ edition; $p p$. 1588-1608. WB Saunders, Philadelphia, 2004); «Wirksamkeit der azellulären Impfstoffe besser» ist falsch, da diese ca. $85 \%$ Wirksamkeit zeigen (Textbook of Pediatric Infectious Diseases. $5^{\text {th }}$ edition; pp. 15881608. WB Saunders, Philadelphia, 2004). «In seltenen Fällen zu Krampfanfällen kommen» - gemeint ist wohl in den ersten Tagen nach Impfung. Dies betrifft v.a. die früher verwendeten Ganzkeimimpfstoffe und nachgewiesenermassen auch nur harmlose Fieberkrämpfe und ist zudem sehr selten, ca. 6-9 pro 100000 Impfdosen! (N Engl J Med 2001;345:656-661). Nach azellulärer Impfung sind es nur 2 Fälle pro 100000 , ohne bewiesenen ursächlichen Zusammenhang (Arch Pediatr Adolesc Med. 1996;150: 457-60). Auch sind nach DTPa-Impfungen
Krampfanfälle nicht häufiger als nach alleiniger DT-Impfung (jeweils 1,1\% während 2,5 Jahren Nachbeobachtungsdauer) (Dev Biol Stand 1997;89:83-89). «Starke Verdachtsmomente für Zusammenhang zwischen Pertussisimpfung und späteren allergischen Erkrankungen» ist eindeutig falsch und mehrfach widerlegt (z.B. Arch Dis Child. 2005 Jun;90:567-73 oder BMJ 2004;17:925-6).

- Auch der Abschnitt «Wegweiser für den persönlichen Impfentscheid» bleibt nicht von Unstimmigkeiten verschont. «Impftermin bei Frühgeborenen auf der Basis des ursprünglichen Geburtstermins» - das widerspricht der biologischen Realität: Frühgeborene haben ein höheres Komplikationsrisiko und einen geringeren Nestschutz als Reifgeborene (da verkürzte Schwangerschaft!); der Abbau des Nestschutzes beginnt mit der Geburt (= Abnabelung), unabhängig von der Reife! Deshalb sollten Frühgeborene wie die Reifgeborenen im chronologischen (!) Alter von 2 Monaten nach Geburt geimpft werden. Zudem: Impfindikation «wenn Eltern sich nicht in der Lage sehen, ein Kind während mehrerer Wochen durch die Krankheit zu begleiten», dies weckt ein schlechtes Gewissen bei den Eltern (quasi Impfung als Notlösung dann, wenn Eltern nicht in der Lage sind ...). «Starke Komplikationen als Kontraindikation für weitere Impfungen» ist $\mathrm{zu}$ pauschal formuliert; lediglich bei allergischen Reaktionen auf Impfstoffbestandteile und «Enzephalopathie innerhalb von 7 Tagen nach früherer Impfung» gelten als Kontraindikationen. Beide Entitäten sind extrem selten. Auch für die Empfehlung zur Zurückhaltung bei «stark allergisch veranlagten Eltern» gibt es keine Evidenz.

Allein auf die oben angeführten Tatsachen gestützt, kann festgehalten werden, dass die Broschüre «Impfen - Grundlagen für einen persönlichen Impfentscheid» (6. Auflage) weder neutral noch vor allem bezüglich medizinischer Impfinformationen korrekt ist. Der Konsument kann leider aufgrund der Lektüre der Broschüre keine informierte Wahl treffen, da falsche oder unstimmige Informationen weitergegeben und falsche Rückschlüsse gezogen werden.

Prof. Dr. med. David Nadal Präsident Schweizerische Gesellschaft für Infektiologie SSI c/o Abt. für Infektiologie, Universitäts-Kinderkliniken Zürich Steinwiesstrasse 75 8032 Zürich 


\section{Pädiatrische Infektiologiegruppe Schweiz (PIGS)}

Wir haben für unseren Kommentar das Kapitel über «Hib-Hirnhautentzündung und Hib-Kehlkopfentzündung» (Seite 40-41) gewählt. Dieses Kapitel enthält weder eine sachlich ausgeglichene noch eine neutrale Darstellung der Daten, was der formulierten Zielsetzung des Ratgebers nämlich dem Leser die kritischen Informationen für einen persönlichen Impfentscheid anzubieten - keineswegs entspricht.

Die Autoren beschreiben, dass vor Einführung der Hib-Impfung in der Schweiz jährlich 100 Kinder an einer Hib-Meningitis, 50 an einer Epiglottitis erkrankten. Dies entspricht den Daten des Bundesamtes für Gesundheit (BAG) bis 1990 (Supplementum VII). Dann schreiben sie: «Nach der Einführung der Impfung im Jahr 1990 sank die Zahl der Erkrankungen bis zum Jahr 2000 auf ca. 10 pro Jahr, um in der Folge bis 2005 wieder auf 64 anzusteigen. Die Nachhaltigkeit der Impfung ist demnach noch offen.» Liest man die dazu zitierten BAG-Meldedaten nach, so findet man, dass zwischen 2000 und 2005 tatsächlich jährlich etwa 60 invasive H.-influenzae-Infektionen gemeldet wurden. Es handelt sich dabei aber um Infektionen aller Altersgruppen (nicht nur der Kinder), verursacht durch alle H.-influenzaeStämme (nicht nur Typ b). Weder bei Kindern $<5$ Jahre (2 bis 13 Fälle pro Jahr) noch in der Gesamtbevölkerung kam es zu einer Zunahme der gemeldeten Fälle. Der Anstieg von 10 auf 64 ist aus der Luft gegriffen. Die fehlende «Nachhaltigkeit» wird mit nachweislich falsch zitierten Zahlen belegt. Diese Falschaussage hinterlässt beim Leser den Eindruck, dass 15 Jahre nach Einführung bereits die Hälfte des Effekts der Impfung wieder verlorengegangen sei, was keineswegs der Fall ist. Eine ausgewogene Information würde eher erwähnen, dass die Einführung der Hib-Impfung vor 15 Jahren bisher - konservativ geschätzt - 1200 Kinder in der Schweiz vor einer bakteriellen Meningitis bewahrt hat und dass bisher kein Trend zur Häufigkeitszunahme durch andere Erreger besteht.

Weiter unten steht: «Ohne Wirkung ist die Impfung auch gegen andere Typen von Haemophilus-influenzae-Bakterien, die in den letzten Jahren zunehmend als Ursache schwerer Erkrankungen identifiziert werden.» Zur Untermauerung dieser Aussage zitieren die Autoren zum einen eine Studie an 2 Patienten (!) aus den USA und eine Studie aus Brasilien (!), deren Bedeutung für die Schweiz fraglich ist. Zum anderen zitieren sie die für die Schweiz relevanteren Überwachungsdaten aus Deutschland. Die genaue Betrachtung derselben zeigt aber, dass es dort zwischen 1998 und 2004 eben gerade nicht $\mathrm{zu}$ einer Zunahme an schweren Nicht-Hib-Erkrankungen gekommen ist (www. esped.uni-duesseldorf.de). Auch hier wird zweifellos und eindeutig falsch zitiert. Dies ist bedauerlich, denn die Möglichkeit einer Serotypenverschiebung nach Einführung der Impfung ist ein ernstzunehmendes mögliches Szenario. Gerade deshalb wird in der Schweiz die Epidemiologie der invasiven Haemophilusinfluenzae-Infektionen überwacht, und ein solcher Trend wird bisher nicht beobachtet. Anstelle dieser für Eltern wichtigen Information in einer ausgewogenen Berichterstattung wird im Ratgeber leider einseitig informiert. Diese unvollständige und einseitige Information kann bei Eltern ungerechtfertigte Skepsis gegenüber der Impfung schüren. Wie bereits in früheren Auflagen des Ratgebers halten die Autoren an der vermeintlichen Assoziation zwischen der Hib-Impfung und der Zunahme des Diabetes mellitus Typ I in westlichen Ländern fest. Sie suggerieren, dass das Risiko eines Kindes, an Diabetes zu erkranken, eine Zehnerpotenz höher sein könne als das Risiko, an einer Hib-Infektion zu erkranken. Die zitierte Aussage, «dass möglicherweise eines von 1700 Kindern als Folge mehrmaliger Hib-Impfungen an jugendlichem Diabetes erkrankte», ist wiederum falsch, sie findet sich in der zitierten Publikation keineswegs. Im Gegenteil: Die Autoren der zitierten Studie kamen zum Schluss, dass keine Evidenz für einen solchen Zusammenhang gefunden werden konnte. Der vom Ratgeber zitierte Kurzbericht der «renommierten» Fachzeitschrift «arznei-telegramm» (eine Kleinstzeitschrift aus Deutschland) erwähnt und interpretiert lediglich ausgewählte Studien Dritter. Zudem stammt diese Referenz aus dem Jahr 1999, während verschiedene nach 1999 in anerkannten Journals publizierte Originalarbeiten (Pediatrics 2001;108:E112; Pediatr Infect Dis J 2002;21:568; N Engl J Med 2004;350:1398-404) keinen Zusammenhang zwischen Hib-Impfung und Diabetes-Risiko fanden. Der Leser (Konsument) erhält wiederum eine einseitige und interpretierte Teilinformation anstelle einer aktuellen und sachlich neutralen Zusammenfassung der vorhandenen Daten.

Schliesslich wird der Eindruck erweckt, dass Stillen einen relevanten Schutzfaktor gegen invasive Hib-Infektionen darstellt. Die Bedeutung des Stillens ist unbestritten. Es ist aufgrund der verfügbaren Literatur dennoch unklar, ob Stillen das Hib-Erkrankungsrisiko von Säuglingen und Kleinkindern verringert. Neuere Studien aus Spanien und Polen (J Infect 2004;48:320; 
Eur J Epidemiol 2003;18:917) fanden keinen solchen Effekt. Die im Ratgeber zitierte Arbeit aus Schweden weist bei genauer Lektüre einen protektiven Effekt nach 5-10 Jahren nach, nicht aber im Kleinkindesalter, wenn Schutz vor Hib-Infektionen wirklich nötig ist. Der Ratgeber argumentiert nochmals mit Halbwahrheiten und zieht damit den Nutzen der Impfung in Zweifel. Nachgewiesenermassen treten invasive Hib-Infektionen bei ungeimpften Kindern schon im ersten Lebensjahr auf, die Hib-Meningitiden erreichen die höchste Inzidenz im Alter vor 12 Monaten (BAG Supplementum VII; CMAJ, 2005;172:53), weshalb empfohlen wird, die Impfung im ersten Lebensjahr zu verabreichen.

Zusammenfassend halten wir nach Durchsicht dieses Kapitels fest, dass die Information zum Thema unvollständig und einseitig ist. Die angegebene Literatur ist einseitig gewählt und eindeutig falsch zitiert, was den Leser irreführt und ihm eine nicht vorhandene sachliche Absicherung vortäuscht. Die fehlende sachliche und neutrale Darstellung der aktuellen Daten erlaubt dem Leser nicht, sich ein eigenes Urteil bilden zu können. Gerade der individuelle Entscheid über eine Impfung aufgrund einer kritischen Auseinandersetzung mit dem Thema wäre ja das Ziel eines solchen Ratgebers.

PD Dr. med. Christoph Berger Präsident Pädiatrische Infektiologiegruppe Schweiz (PIGS) LA Infektiologie und Spitalhygiene Universitäts-Kinderkliniken Zürich Steinwiesstr. 75 8032 Zürich 